\title{
EVALUATION OF INDIVIDUAL MONITORING IN MIXED NEUTRON/PHOTON FIELDS: MID-TERM RESULTS FROM THE EVIDOS PROJECT
}

\author{
F. Vanhavere ${ }^{1, *}$, D. Bartlett ${ }^{2}$, T. Bolognese-Milsztajn ${ }^{3}$, M. Boschung ${ }^{4}$, M. Coeck ${ }^{1}$, G. Curzio ${ }^{5}$, \\ F. d'Errico ${ }^{5}$, A. Fiechtner ${ }^{4}$, J. Kyllönen ${ }^{6}$, V. Lacoste ${ }^{3}$, T. Lahaye ${ }^{3}$, L. Lindborg ${ }^{6}$, M. Luszik-Bhadra ${ }^{7}$, \\ C. Molinos ${ }^{2}$, H. Muller ${ }^{3}$, M. Reginatto ${ }^{7}$, H. Schuhmacher ${ }^{7}$ and R. Tanner ${ }^{2}$ \\ ${ }^{1}$ Studiecentrum voor Kernenergie - Centre d'étude de l'énergie nucléaire, B-2400 Mol, Belgium \\ ${ }^{2}$ National Radiological Protection Board, Chilton, Didcot OX11 0RQ, UK \\ ${ }^{3}$ Institut de Radioprotection et de Sûreté Nucléaire, F-92265 Fontenay-aux-Roses, France \\ ${ }^{4}$ Paul Scherrer Institut, CH-5232 Villigen, Switzerland \\ ${ }^{5}$ Dipartimento di Ingegneria Meccanica, Nucleare e della Produzione, I-56126 Pisa, Italy \\ ${ }^{6}$ Statens strålskyddsinstitut, SE-171-16 Stockholm, Sweden \\ ${ }^{7}$ Physikalisch-Technische Bundesanstalt, D-38116 Braunschweig, Germany
}

\begin{abstract}
EVIDOS is an EC sponsored project that aims at an evaluation and improvement of radiation protection dosimetry in mixed neutron/photon fields. This is performed through spectrometric and dosimetric investigations during different measurement campaigns in representative workplaces of the nuclear industry. The performance of routine and, in particular, novel personal dosemeters and survey instruments is tested in selected workplace fields. Reference values for the dose equivalent quantities, $H^{*}(10)$ and $H_{\mathrm{p}}(10)$ and the effective dose $E$, are determined using different spectrometers that provide the energy distribution of the neutron fluence and using newly developed devices that determine the energy and directional distribution of the neutron fluence. The EVIDOS project has passed the mid-term, and three measurement campaigns have been performed. This paper will give an overview and some new results from the third campaign that was held in Mol (Belgium), around the research reactor VENUS and in the MOX producing plant of Belgonucléaire.
\end{abstract}

\section{INTRODUCTION}

Radiation protection dosimetry in mixed neutron/ photon fields is still far less established than for photon radiation alone. In practice, passive devices are used with high dose threshold and not ideal energy characteristics. Also, area monitor readings can deviate significantly from the radiation protection quantities. Still, $>60,000$ workers in the EU and Switzerland need to be monitored for neutrons.

Within its 5th Framework Programme, the EC is funding the project EVIDOS which will perform an evaluation of individual dosimetry in mixed neutron and photon radiation fields at workplaces of the nuclear fuel cycle, with special regard to neutrons. Recently, some active devices became commercially available for neutron dosimetry ${ }^{(1,2)}$. One of the primary aims of the project is establishing whether innovative electronic dosemeters with direct reading allow improved determination of personal dose equivalent.

The following tasks are carried out:

(i) determination of the energy and angle distribution of the neutron fluence;

${ }^{*}$ Corresponding author: fvanhave@sckcen.be (ii) derivation of the values of radiation protection quantities;

(iii) determination of the readings of routine and innovative personal dosemeters and of area monitors;

(iv) comparison between dosemeter readings and radiation protection quantities.

The measurements took place in selected mixed-radiation environments representative of the fields encountered in the nuclear fuel cycle. These environments permit a thorough testing of the dosemeters since they differ widely in terms of dose rates, neutron/photon relative intensity, energy distributions, and also temperature, pressure, humidity, acoustic noise, vibration, electromagnetic fields, and so on.

A first exercise was performed at the IRSN simulated workplace facility CANEL/T400 and at the thermal neutron source SIGMA (campaign 0). The purpose of the exercise was to verify the behaviour of all instruments in a well-established reference field similar to some of those encountered at workplaces. Following this, two measurement campaigns have been performed: campaign number 1 was carried out in Krümmel, Germany, inside a boiling water reactor building and around a storage cask 


\section{F. VANHAVERE ET AL.}

containing spent fuel elements. Campaign number 2 was carried out in Mol, Belgium, at the Belgonucléaire MOX fuel element manufacturing plant and at the $\mathrm{SCK} \cdot \mathrm{CEN}$ VENUS research reactor. One or two additional campaigns are scheduled: inside a pressurised water reactor building and around a transport cask in Ringhals, Sweden and/or at a nuclear facility in the UK.

\section{MATERIALS AND METHODS}

Because no current dosemeter gives correct results in all neutron fields, reference values have to be derived from spectrometry and fluence-to-dose equivalent conversion coefficients. In the case of neutron radiation, angle-differential fluence distributions were determined along with the energy-differential values. These were needed to correlate isotropic quantities, such as ambient dose equivalent and total fluence, to non-isotropic ones, such as personal dose equivalent and effective dose.

Reference field spectrometry is primarily performed with a Bonner-sphere system ${ }^{(3)}$ for the entire energy range. The measurements of the doubledifferential (energy and direction) neutron fluence are performed with novel instruments based on SDD-spectrometers ${ }^{(4)}$ and Si-diodes ${ }^{(5)}$.

The IRSN Bonner sphere spectrometer is used for reference spectrometry. It consists of a ${ }^{3} \mathrm{He}$ filled proportional counter and 12 polyethylene spheres. The five smallest spheres are used bare and also with a cadmium shield.

In this paper only results from the directional spectrometer based on the Si-diodes are reported. This directional spectrometer consists of six detector capsules - each containing a stack of four silicon detectors-mounted onto the surface of a $30 \mathrm{~cm}$ diameter polyethylene sphere, and electronics to amplify and record the pulse height spectra of all detectors. The pulse height spectra measured in workplace fields are analysed using unfolding codes with respect to energy and direction both for neutrons and photons. Values of ambient dose equivalent and personal dose equivalent $H_{\mathrm{p}}(10)$ for the main direction of incidence are derived by multiplying the fluence distributions by the corresponding fluence-to-dose conversion coefficients.

In every workplace field, all available personal neutron dosemeters are exposed. Both commercial types of active neutron dosemeters (Aloka PDM-313, Saphydose-n, EPDN2, EPDN, BD-PND, BDT) and pre-commercial types (PTB DOS2002, PSI DISN) are used. One novel reference method for $H_{\mathrm{p}}(10)$, DIMNP HpSLAB, and two novel area monitors (NRPB hybrid area monitor, Sievert instrument) are tested as well. Also conventional area monitors for neutrons and photons as well as passive individual dosemeters (CR-39, PADC) for routine monitoring are used.

The measurement results of all these devices are compared with the reference values of the appropriate radiation protection quantities. To evaluate the results, the response of these instruments are needed for the entire range of energies and angles of incidence. A method was developed to determine the response of a neutron dosemeter, combining response data from measurements with monoenergetic neutron fields and response data from measurements in fields with broad energy distributions. This novel method has been applied for the dosemeters used within EVIDOS, and has been described by Reginatto et al. ${ }^{(6)}$

\section{RESULTS}

\section{C0: Cadarache measurement campaign}

Two fields were used to irradiate the dosemeters: SIGMA $^{(7)}$, where almost half of the ambient dose equivalent arises from thermal neutrons, and CANEL $^{(8)}$ with a maximum contribution to the dose equivalent of a few hundred $\mathrm{keV}$ and additional significant contributions of thermal and intermediate energy neutrons.

The behaviour of the personal and area dosemeters in CANEL and SIGMA has been described previously ${ }^{(9)}$. At CANEL and SIGMA, the dosemeters Aloka PDM-313 and Siemens EPD N2 showed over-readings by more than a factor of two compared with the reference values, while others (Saphydose- $n$, DOS-2002, PADC track detectors from NRPB and devices based on superheated drop detectors like HpSLAB and BD-PND) showed deviations of $<30 \%$. The Siemens EPD N, a dosemeter which is intended only for the measurement of thermal and intermediate energy neutrons, responded well to the thermal part of the spectrum at SIGMA but showed almost zero readings at CANEL.

\section{C1: Krümmel measurement campaign}

In the Krümmel nuclear power plant measurements were performed at four workplaces: a position in the control rod room underneath the reactor ('reactor SAR'), another position near the top of the reactor ('reactor top') and two places near a cask containing used fuel ('cask midline' and 'cask side'). The neutron fluence measured with the Bonner sphere spectrometer gave deviations of up to $30 \%$ compared to the integrated fluences obtained by the directional spectrometer. The spectra at the cask were quite hard, with a main fluence contribution at a few hundred $\mathrm{keV}$, while the spectra at the reactor contained a considerable amount of thermal and intermediate energy neutrons. 
Most of the electronic personal dosemeters show conservative (too high) readings in the fields investigated at the Krümmel reactor. A full discussion of the results at Krümmel is given by Luszik-Bhadra et al. ${ }^{(10,11)}$

\section{C2: Mol measurement campaign}

In Mol, the measurements for the C2 campaign were performed at two facilities. At the VENUS Research Reactor one location was chosen where personnel need to read a gauge next to the reactor containment (point F) and another in the control room (point C). At the Belgonucléaire fuel processing plant, four positions were chosen: bare MOX rods (point 1), MOX rods in a rack with (point 2A) and without shielding (point 2B) and inside a storage room (point 3).

In Figures 1 and 2 the spectra measured with the Bonner sphere system are shown for two locations: points $2 \mathrm{~A}$ and 3 at Belgonucléaire. The unfolding was done in two steps ${ }^{(12)}$ : the Bayesian parameter estimation program NUBAY was used to derive an initial estimate of the spectrum (as well as estimates of integral quantities), and the estimate of the spectrum was then used as default spectrum for the iterative unfolding code GRAVEL. These spectra yielded the reference values of $H^{*}(10)$ that are shown in Table 1 . The overall uncertainty includes the standard deviation determined with NUBAY, a $3 \%$ systematic uncertainty from the response functions and the deviation between the NUBAY and GRAVEL results.

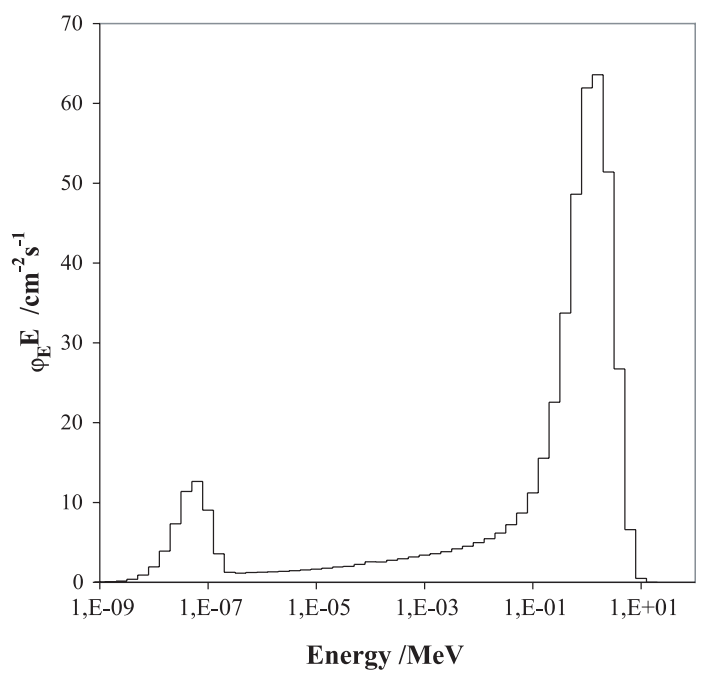

Figure 1. Spectral neutron fluence (lethargy representation) at unshielded MOX rods in a rack ( $\mathrm{BN}$ point $2 \mathrm{~A})$.
The differential spectrometer results are still being processed, but the ratio $H_{\mathrm{p}}(10) / H^{*}(10)$ is already available and is shown in Table 1. For these values, four different unfolding procedures are used as described by Luszik-Bhadra et al. ${ }^{(11)}$

The MOX rods in point $2 \mathrm{~A}$ result in a very hard spectrum, since hardly any shielding is present around this rack. The measurement point was in the middle of the $4 \mathrm{~m}$ long rack, with little background neutrons present. The $H_{\mathrm{p}}(10) / H^{*}(10)$ value of 0.80 shows that the directional distribution is quite uniform from the front direction. In the storage room different racks filled with MOX rods are present, spread out over a larger area. So the $H_{\mathrm{p}}(10) / H^{*}(10)$ value of 0.54 is lower than at the single rack. There is also more shielding and neutron scattering, resulting in the presence of a thermal neutron peak (Figure 2).

The photon dose rate results, measured with the FHT ionisation chamber, showed that neutron doses

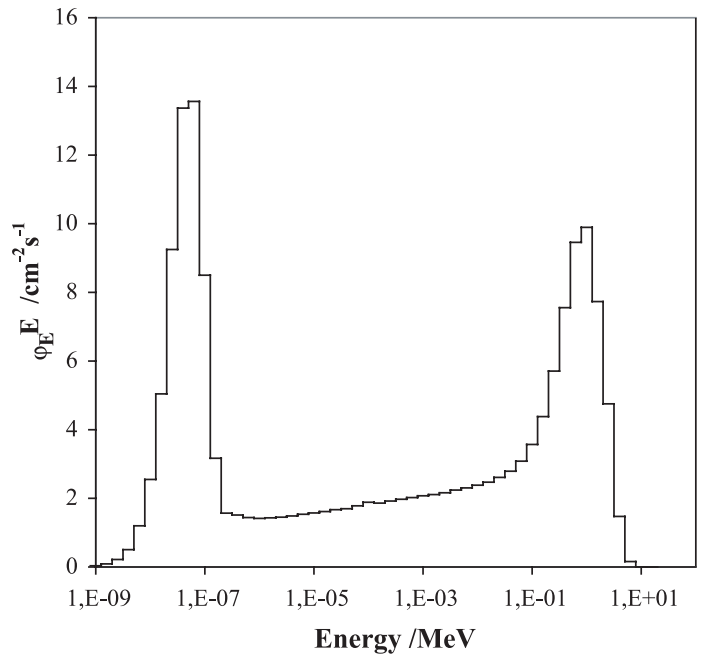

Figure 2. Spectral neutron fluence (lethargy representation) at the MOX rod storage room (BN point 3 ).

Table 1. Reference values for two points of the Mol campaign for the neutron component [measured with Bonner spheres (BS) and the directional spectrometer (DS)] and for the photon component (measured with the FHT 191 ionisation chamber).

\begin{tabular}{lccc}
\hline & $\begin{array}{c}\text { BS } \dot{H}^{*}(10) \\
\text { neutron } \\
\left(\mu \mathrm{S} v \mathrm{~h}^{-1}\right)\end{array}$ & $\begin{array}{c}\text { DS } H_{\mathrm{p}}(10) / \\
H^{*}(10) \\
\text { neutron }\end{array}$ & $\begin{array}{c}\text { FHT 191 } \dot{H}^{*}(10) \\
\text { photon } \\
\left(\mu \mathrm{Sv} \mathrm{h}^{-1}\right)\end{array}$ \\
\hline BN Point 2A & $209(7)$ & $0.80(2)$ & 28.3 \\
BN Point 3 & $32.0(1.1)$ & $0.54(2)$ & 12.2 \\
\hline
\end{tabular}

Values in parentheses denote standard uncertainties 


\section{F. VANHAVERE ET AL.}

are the most important component of the total dose rate at the chosen locations (between 70 and 90\%).

The area monitor results are given in Table 2, together with the total uncertainty derived from that of the calibration and counting statistics. Almost all area monitors underestimate the reference values for both points, but most results stay within $30 \%$ of the reference value. Most area monitors have a minimum in the response at $\sim 500 \mathrm{keV}$ (slightly different for the different types) up to $1 \mathrm{MeV}$ or higher (depending on calibration). In cases with a low part of scattered neutrons and spectra centered at $\sim 1 \mathrm{MeV}$, like at these two points at the Belgonucléaire facility, such an underresponse seems explainable.

In Figures 3 and 4 the results of the personal dosemeters are given, together with the reference values of $H^{*}(10)$ and $H_{\mathrm{p}}(10)$. The latter have been derived by multiplying the values of $H^{*}(10)$ from the Bonner sphere measurements by the ratios $H_{\mathrm{p}}(10) / H^{*}(10)$ from the directional spectrometer. The response of the personal dosemeters is different in both workplaces. The BD-PND bubble detectors overrespond (less than factor 2) in both

Table 2. Area monitor results for two points at the Mol campaign (including standard uncertainties).

\begin{tabular}{lcc}
\hline$H^{*}(10)$ response & Point 2A, BN & Point 3, BN \\
\hline Berthold LB 6411 & $0.88(6)$ & $0.62(5)$ \\
Harwell N91 & $0.88(5)$ & $0.83(5)$ \\
Studsvik 2202D & $0.79(4)$ & $0.76(5)$ \\
Wendi-2 & $0.89(5)$ & $0.90(6)$ \\
Cramal 21 & $0.67(5)$ & $0.78(6)$ \\
\hline
\end{tabular}

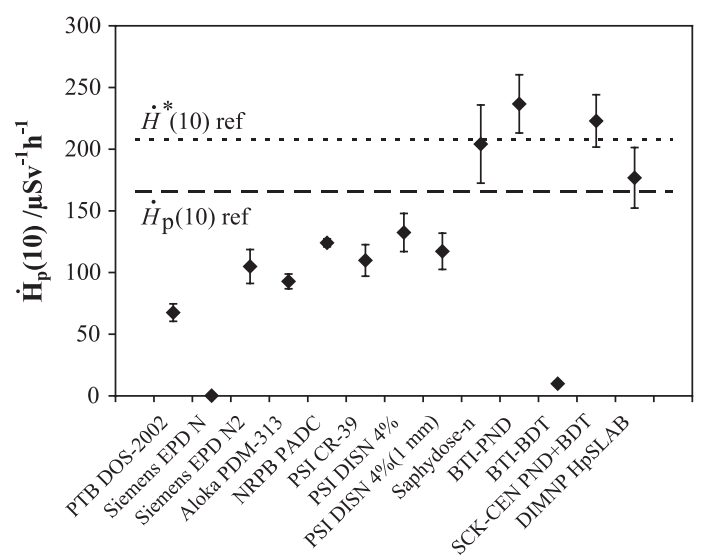

Figure 3. Personal dosemeter readings at point $2 \mathrm{~A}$ in Belgonucléaire (Non-shielded MOX-rack). The dashed and dotted lines indicate the reference values for $\dot{H}_{\mathrm{p}}(10)$ and $\dot{H}^{*}(10)$, respectively. fields. These bubble detectors are the dosemeters used locally for personal dosimetry ${ }^{(13)}$. Also the Saphydose- $n$ shows a small overestimation in both fields. The dosemeters based on CR-39/ PADC showed in general very good agreement with the $H_{\mathrm{p}}(10)$ values. The Aloka PDM-313 and the EPDN2 result in an underestimation at point 2A. The performance of the EPDN2 is better at the mixed field at point 3, while the Aloka PDM-313 overestimates. The PTB DOS-2002 underestimates by roughly a factor of 2 in the MOX fields. It is important to note that the uncertainties of the instruments' readings do not include the contributions from the energy and direction dependence of their response.

\section{CONCLUSION AND OUTLOOK}

The EVIDOS program has currently passed its midterm. A wealth of data has already been collected and is being analysed. Preliminary results show a large variation of response of the radiation protection instruments in the different workplace fields. Although some behave reasonable well in certain fields, the limitations of the dosemeters must be realised, and a field specific correction factor might be necessary. Important is the knowledge of energy and direction distribution of neutrons for the investigated fields. Such additional information can be obtained by the directional spectrometer, but also in simpler terms by measurements with superheated drop detectors and PADC track detectors

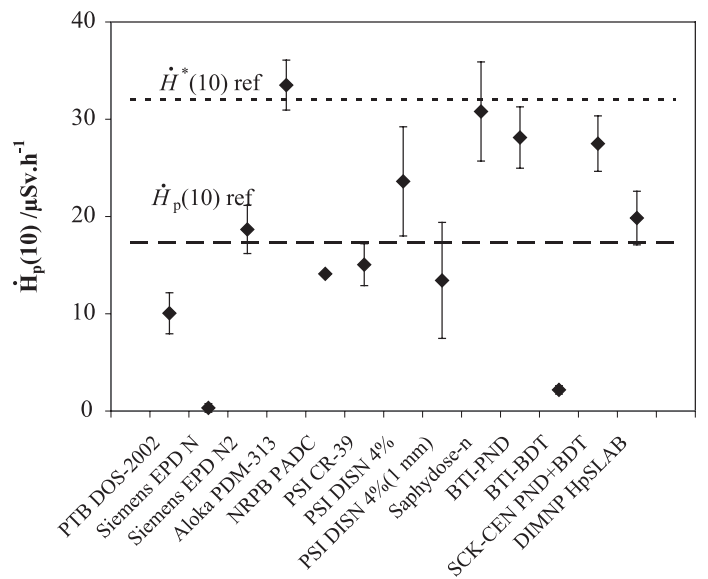

Figure 4. Personal dosemeter readings at point 3 in Belgonucléaire (storage room). The dashed and dotted lines indicate the reference values for $\dot{H}_{\mathrm{p}}(10)$ and $\dot{H}^{*}(10)$, respectively. 
mounted in different directions on the sides of the phantom.

\section{ACKNOWLEDGEMENTS}

The authors wish to express their gratitude to the technical and scientific staff at Cadarache, Krümmel and Mol who helped during the campaigns. This research is partly funded by the European Commission under the auspices of the Euratom 5th Framework Programme: Nuclear Energy, 1998-2002, Contract No FIKR-CT-2001-00175.

\section{REFERENCES}

1. Luszik-Bhadra, M. Electronic personal dosemeters: the solution to problems of individual monitoring in mixed neutron/photon fields? Radiat. Prot. Dosim. 110(1-4), 747-752 (2004).

2. d'Errico, F., Luszik-Bhadra, M. and Lahaye, T. State of the art of electronic personal dosimeters for neutrons. Nucl. Instrum. Methods Phys. Res. A 505(1-2), 411-414 (2003).

3. Lacoste, V., Gressier, V., Pochat, J.L., Fernández, F., Bakali, M. and Bouassoule, T. Characterization of Bonner sphere systems at mono-energetic and thermal neutron fields. Radiat. Prot. Dosim. 110(1-4), 529-532 (2004).

4. d'Errico, F., Giusti, V., Reginatto, M. and Wiegel, B. A telescope design directional neutron spectrometer. Radiat. Prot. Dosim. 110(1-4), 533-537 (2004).

5. Luszik-Bhadra, M., Reginatto, M. and Lacoste, V. Measurement of energy and directional distribution of neutron and photon fluences at workplace fields. Radiat. Prot. Dosim. 110(1-4), 237-241 (2004).
6. Reginatto M., Böttger R., d'Errico F., Fiechtner A., Friedrich H., Lacoste V., Lahaye T., Luszik-Bhadra M. and Vanhavere F. Dose equivalent response of neutron dosemeters. In: Proceedings of IRPA 11, 23-28 May, Madrid, Spain (2004).

7. Lacoste, V., Gressier, V., Muller, H. and Lebreton, L. Characterization of the IRSN graphite moderated americium-beryllium neutron field. Radiat. Prot. Dosim. 110(1-4), 135-139 (2004).

8. Gressier, V. et al. Characterization of the IRSN CANEL/T400 facility producing realistic neutron fields for calibration and test purposes. Radiat. Prot. Dosim. 110(1-4), 523-527 (2004).

9. Bolognese-Milsztajn, T. et al. Individual monitoring in workplaces with mixed neutron/photon radiation. Radiat. Prot. Dosim. 110(1-4), 753-758 (2004).

10. Luszik-Bhadra, M. et al. EVIDOS: optimisation of individual monitoring in mixed neutron/photon fields at workplaces of the nuclear fuel cycle. In: Proceedings of the 4th ISOE European workshop on occupational exposure management at NPPs, 24-26 March, Lyon, France (2004). Published on the website http://isoe. cepn.asso.fr/.

11. Luszik-Bhadra, M. et al. Electronic neutron personal dosemeters: their performance in mixed radiation fields in Nuclear Power Plants. Radiat. Prot. Dosim. 120(1-4), 378-382 (2006).

12. Lacoste, V., Muller, H., Bolognese, T. and Reginatto, M. Neutron Spectrometry with Bonner spheres at the Belgonucleaire MOX fuel processing plant, IRSN Report, IRSN/DRPH/SDE - Report 2004-34 (2004).

13. Olaerts R., Kockerols P., Renard A., Rosenstock W., Köble T. and Vanhavere F. Determination of neutron spectra in a MOX plant for the qualification of the BD-PND Bubble Detector. Health Phys. 2(77), 200-206 (1999). 